\title{
Better Results for Trigonometrically Convex Functions via Hölder-İscan and Improved Power-Mean Inequalities
}

\author{
Mahir Kadakal ${ }^{1}$ \\ ${ }^{1}$ Department of Mathematics, Faculty of Science and Arts, Giresun University, Giresun, Turkey
}

\section{Article Info}

Keywords: Convex function, Trigonometrically convex function, HölderIscan integral inequality, Improved power-mean integral inequality 2010 AMS: 26A51, 26D10, $26 D 15$.

Received: 29 May 2019

Accepted: 25 February 2020

Available online: 25 March 2020

\begin{abstract}
In this paper, using Hölder-İscan and improved power-mean integral inequalities and together with an integral identity, we obtain Hadamard type inequalities for functions whose second derivatives in absolute value at certain power are trigonometrically convex functions. In addition, we prove that our results give better approach than previous results.
\end{abstract}

\section{Introduction}

Throughout the paper $I$ is a non-empty interval in $\mathbb{R}$.

Definition 1.1. A function $f: I \rightarrow \mathbb{R}$ is said to be convex if the inequality

$$
f(t x+(1-t) y) \leq t f(x)+(1-t) f(y)
$$

is valid for all $x, y \in I$ and $t \in[0,1]$. If this inequality reverses, then $f$ is said to be concave on interval $I \neq \emptyset$.

Convexity theory has appeared as a powerful technique to study a wide class of related problems in pure and applied sciences. See articles $[2,4,9-12]$ and the references therein.

Let $f:[a, b] \rightarrow \mathbb{R}$ be a convex function, then the inequality

$$
f\left(\frac{a+b}{2}\right) \leq \frac{1}{b-a} \int_{a}^{b} f(x) d x \leq \frac{f(a)+f(b)}{2}
$$

is known as the Hermite-Hadamard inequality (for more information, see [5] ). Since then, some refinements of the Hermite-Hadamard inequality for convex functions have been obtained $[2,3,13,15]$.

Definition 1.2 ( [14]). Let $h:(0,1) \subset J \rightarrow \mathbb{R}$ be a non-negative function, $h \neq 0$. We say that $f: I \rightarrow \mathbb{R}$ is an $h$-convex function, or that $f$ belongs to the class $S X(h, I)$, if $f$ is non-negative and for all $x, y \in I, \alpha \in(0,1)$ we have

$$
f(\alpha x+(1-\alpha) y) \leq h(\alpha) f(x)+h(1-\alpha) f(y) .
$$

If this inequality is reversed, then $f$ is said to be h-concave, i.e. $f \in S V(h, I)$.

Definition 1.3 ( [7]). A non-negative function $f: I \rightarrow \mathbb{R}$ is called trigonometrically convex function on interval $[a, b]$, if for each $x, y \in[a, b]$ and $t \in[0,1]$,

$$
f(t x+(1-t) y) \leq\left(\sin \frac{\pi t}{2}\right) f(x)+\left(\cos \frac{\pi t}{2}\right) f(y) .
$$


Denoted by $T C(I)$ the class of all trigonometrically convex functions on interval $I$. Every non-negative convex function is trigonometrically convex and every trigonometrically convex function is $h$-convex with $h(t)=\frac{\pi t}{2}$.

A refinement of Hölder integral inequality better approach than Hölder integral inequality can be given as follows:

Theorem 1.4 (Hölder-İşcan Integral Inequality [6]). Let $p>1$ and $\frac{1}{p}+\frac{1}{q}=1$. If $f$ and $g$ are real functions defined on $[a, b]$ and if $|f|^{p},|g|^{q}$ are integrable functions on interval $[a, b]$ then

$$
\begin{aligned}
\int_{a}^{b}|f(x) g(x)| d x & \leq \frac{1}{b-a}\left\{\left(\int_{a}^{b}(b-x)|f(x)|^{p} d x\right)^{\frac{1}{p}}\left(\int_{a}^{b}(b-x)|g(x)|^{q} d x\right)^{\frac{1}{q}}\right. \\
& \left.\left(\int_{a}^{b}(x-a)|f(x)|^{p} d x\right)^{\frac{1}{p}}\left(\int_{a}^{b}(x-a)|g(x)|^{q} d x\right)^{\frac{1}{q}}\right\} .
\end{aligned}
$$

Improwed power-mean integral inequality as a result of the Hölder-İscan integral inequality can be given as follows:

Theorem 1.5 (Improved power-mean integral inequality [8]). Let $q \geq 1$. If $f$ and $g$ are real functions defined on $[a, b]$ and if $|f|,|f||g|^{q}$ are integrable functions on $[a, b]$ then

$$
\begin{aligned}
\int_{a}^{b}|f(x) g(x)| d x & \leq \frac{1}{b-a}\left\{\left(\int_{a}^{b}(b-x)|f(x)| d x\right)^{1-\frac{1}{q}}\left(\int_{a}^{b}(b-x)|f(x)||g(x)|^{q} d x\right)^{\frac{1}{q}}\right. \\
& \left.+\left(\int_{a}^{b}(x-a)|f(x)| d x\right)^{1-\frac{1}{q}}\left(\int_{a}^{b}(x-a)|f(x)||g(x)|^{q} d x\right)^{\frac{1}{q}}\right\} .
\end{aligned}
$$

Definition 1.6. (Beta Function) The Beta function denoted by $\beta(a, b)$ is defined by

$$
\beta(a, b)=\int_{0}^{1} t^{a-1}(1-t)^{b-1} d t, a, b>0 .
$$

\section{Main results}

In this section, using Hölder-İşcan integral inequality and improved power-mean integral inequality and an integral identity, author obtain a generalization of Hermite-Hadamard type inequalities for functions whose second derivatives in absolute value at certain power are trigonometrically convex functions.

In order to establish some inequalities of Hermite-Hadamard type integral inequalities for trigonometrically convex functions, we will use the following lemma. This lemma can be easily obtained by taking partial integration in the lemma in [1] .

Lemma 2.1. The following equality holds:

$$
\frac{f(a)+f(b)}{2}-\frac{1}{b-a} \int_{a}^{b} f(x) d x=\frac{(b-a)^{2}}{2} \int_{0}^{1}\left(t-t^{2}\right) f^{\prime \prime}(t a+(1-t) b) d t
$$

Theorem 2.2. Let $f: I \rightarrow \mathbb{R}$ be a continuously two times differentiable function, let $a<b$ in I. If the mapping $\left|f^{\prime \prime}\right|$ is trigonometrically convex function on interval $[a, b]$, then the following inequality

$$
\left|\frac{f(a)+f(b)}{2}-\frac{1}{b-a} \int_{a}^{b} f(x) d x\right| \leq(b-a)^{2} \frac{16-4 \pi}{\pi^{3}} A\left(\left|f^{\prime \prime}(a)\right|,\left|f^{\prime \prime}(b)\right|\right)
$$

holds for $t \in[0,1]$, where $A$ is the arithmetic mean and $\frac{1}{p}+\frac{1}{q}=1$.

Proof. Using Lemma 2.1 and inequality

$$
\left|f^{\prime \prime}(t a+(1-t) b)\right| \leq\left(\sin \frac{\pi t}{2}\right)\left|f^{\prime \prime}(a)\right|+\left(\cos \frac{\pi t}{2}\right)\left|f^{\prime \prime}(b)\right|,
$$

we obtain

$$
\begin{aligned}
\left|\frac{f(a)+f(b)}{2}-\frac{1}{b-a} \int_{a}^{b} f(x) d x\right| & \leq \frac{(b-a)^{2}}{2} \int_{0}^{1}|t||1-t|\left|f^{\prime \prime}(t a+(1-t) b)\right| d t \\
& \leq \frac{(b-a)^{2}}{2}\left(\int_{0}^{1} t(1-t)\left|f^{\prime \prime}(t a+(1-t) b)\right| d t\right) \\
& \leq \frac{(b-a)^{2}}{2}\left(\int_{0}^{1} t(1-t)\left[\left(\sin \frac{\pi t}{2}\right)\left|f^{\prime \prime}(a)\right|+\left(\cos \frac{\pi t}{2}\right)\left|f^{\prime \prime}(b)\right|\right] d t\right) \\
& =\frac{(b-a)^{2}}{2}\left[\left(\frac{16-4 \pi}{\pi^{3}}\right)\left|f^{\prime \prime}(a)\right|+\left(\frac{16-4 \pi}{\pi^{3}}\right)\left|f^{\prime \prime}(b)\right|\right] \\
& =(b-a)^{2} \frac{16-4 \pi}{\pi^{3}} A\left(\left|f^{\prime \prime}(a)\right|,\left|f^{\prime \prime}(b)\right|\right)
\end{aligned}
$$

where

$$
\int_{0}^{1} t(1-t) \sin \frac{\pi t}{2} d t=\int_{0}^{1} t(1-t) \cos \frac{\pi t}{2} d t=\frac{16-4 \pi}{\pi^{3}} .
$$

This completes the proof of the theorem. 
Theorem 2.3. Let $f: I \rightarrow \mathbb{R}$ be a continuously two times differentiable function, let $a<b$ in I and assume that $q>1$. If the mapping $\left|f^{\prime \prime}\right|^{q}$ is trigonometrically convex function on interval $[a, b]$, then the following inequality

$$
\left|\frac{f(a)+f(b)}{2}-\frac{1}{b-a} \int_{a}^{b} f(x) d x\right| \leq \frac{(b-a)^{2}}{2}\left(\frac{4}{\pi}\right)^{\frac{1}{q}} \beta^{\frac{1}{p}}(p+1, p+1) A^{\frac{1}{q}}\left(\left|f^{\prime \prime}(a)\right|^{q},\left|f^{\prime \prime}(b)\right|^{q}\right)
$$

holds for $t \in[0,1]$, where $\frac{1}{p}+\frac{1}{q}=1$.

Proof. Using Lemma 2.1, Hölder integral inequality and inequality

$$
\left|f^{\prime \prime}(t a+(1-t) b)\right|^{q} \leq\left(\sin \frac{\pi t}{2}\right)\left|f^{\prime \prime}(a)\right|^{q}+\left(\cos \frac{\pi t}{2}\right)\left|f^{\prime \prime}(b)\right|^{q}
$$

which is the trigonometrically concexity of $\left|f^{\prime \prime}\right|^{q}$, we obtain

$$
\begin{aligned}
\left|\frac{f(a)+f(b)}{2}-\frac{1}{b-a} \int_{a}^{b} f(x) d x\right| & \leq \frac{(b-a)^{2}}{2} \int_{0}^{1}|t||1-t|\left|f^{\prime \prime}(t a+(1-t) b)\right| d t \\
& \leq \frac{(b-a)^{2}}{2}\left(\int_{0}^{1} t^{p}(1-t)^{p} d t\right)^{\frac{1}{p}}\left(\int_{0}^{1}\left|f^{\prime \prime}(t a+(1-t) b)\right|^{q} d t\right)^{\frac{1}{q}} \\
& \leq \frac{(b-a)^{2}}{2}\left(\int_{0}^{1} t^{p}(1-t)^{p} d t\right)^{\frac{1}{p}}\left(\int_{0}^{1}\left[\left(\sin \frac{\pi t}{2}\right)\left|f^{\prime \prime}(a)\right|^{q}+\left(\cos \frac{\pi t}{2}\right)\left|f^{\prime \prime}(b)\right|^{q}\right] d t\right) \\
& =\frac{(b-a)^{2}}{2} \beta^{\frac{1}{p}}(p+1, p+1)\left(\left|f^{\prime \prime}(a)\right|^{q} \frac{2}{\pi}+\left|f^{\prime \prime}(b)\right|^{q} \frac{2}{\pi}\right)^{\frac{1}{q}} \\
& =\frac{(b-a)^{2}}{2}\left(\frac{4}{\pi}\right)^{\frac{1}{q}} \beta^{\frac{1}{p}}(p+1, p+1) A^{\frac{1}{q}}\left(\left|f^{\prime \prime}(a)\right|^{q},\left|f^{\prime \prime}(b)\right|^{q}\right)
\end{aligned}
$$

where

$$
\int_{0}^{1} t^{p}(1-t)^{p} d t=\beta(p+1, p+1)
$$

This completes the proof of the theorem.

Theorem 2.4. Let $f: I \rightarrow \mathbb{R}$ be a continuously two times differentiable function, let $a<b$ in I and assume that $q>1$. If the mapping $\left|f^{\prime \prime}\right|^{q}$ is trigonometrically convex function on interval $[a, b]$, then the following inequality

$$
\begin{aligned}
\left|\frac{f(a)+f(b)}{2}-\frac{1}{b-a} \int_{a}^{b} f(x) d x\right| & \leq \frac{(b-a)^{2}}{2} \beta^{\frac{1}{p}}(p+1, p+2)\left[\left(\frac{2}{\pi}-\frac{4}{\pi^{2}}\right)\left|f^{\prime \prime}(a)\right|^{q}+\left(\frac{4}{\pi^{2}}\right)\left|f^{\prime \prime}(b)\right|^{q}\right]^{\frac{1}{q}} \\
& +\frac{(b-a)^{2}}{2} \beta^{\frac{1}{p}}(p+2, p+1)\left[\frac{4}{\pi^{2}}\left|f^{\prime \prime}(a)\right|^{q}+\left(\frac{2}{\pi}-\frac{4}{\pi^{2}}\right)\left|f^{\prime \prime}(b)\right|^{q}\right]^{\frac{1}{q}}
\end{aligned}
$$

holds for $t \in[0,1]$, where $\frac{1}{p}+\frac{1}{q}=1$.

Proof. Using Lemma 2.1, Hölder-İşcan integral inequality and inequality

$$
\left|f^{\prime \prime}(t a+(1-t) b)\right|^{q} \leq\left(\sin \frac{\pi t}{2}\right)\left|f^{\prime \prime}(a)\right|^{q}+\left(\cos \frac{\pi t}{2}\right)\left|f^{\prime \prime}(b)\right|^{q}
$$

which is the trigonometrically concexity of $\left|f^{\prime \prime}\right|^{q}$, we obtain

$$
\begin{aligned}
\left|\frac{f(a)+f(b)}{2}-\frac{1}{b-a} \int_{a}^{b} f(x) d x\right| & \leq \frac{(b-a)^{2}}{2} \int_{0}^{1}|t||1-t|\left|f^{\prime \prime}(t a+(1-t) b)\right| d t \\
& \leq \frac{(b-a)^{2}}{2}\left(\int_{0}^{1}(1-t) t^{p}(1-t)^{p} d t\right)^{\frac{1}{p}}\left(\int_{0}^{1}(1-t)\left|f^{\prime \prime}(t a+(1-t) b)\right|^{q} d t\right)^{\frac{1}{q}} \\
& +\frac{(b-a)^{2}}{2}\left(\int_{0}^{1} t t^{p}(1-t)^{p} d t\right)^{\frac{1}{p}}\left(\int_{0}^{1} t\left|f^{\prime \prime}(t a+(1-t) b)\right|^{q} d t\right)^{\frac{1}{q}} \\
& \leq \frac{(b-a)^{2}}{2}\left(\int_{0}^{1}(1-t) t^{p}(1-t)^{p} d t\right)^{\frac{1}{p}}\left(\int_{0}^{1}(1-t)\left[\left(\sin \frac{\pi t}{2}\right)\left|f^{\prime \prime}(a)\right|^{q}+\left(\cos \frac{\pi t}{2}\right)\left|f^{\prime \prime}(b)\right|^{q}\right] d t\right)^{\frac{1}{q}} \\
& +\frac{(b-a)^{2}}{2}\left(\int_{0}^{1} t t^{p}(1-t)^{p} d t\right)^{\frac{1}{p}}\left(\int_{0}^{1} t\left[\left(\sin \frac{\pi t}{2}\right)\left|f^{\prime \prime}(a)\right|^{q}+\left(\cos \frac{\pi t}{2}\right)\left|f^{\prime \prime}(b)\right|^{q}\right] d t\right)^{\frac{1}{q}} \\
& =\frac{(b-a)^{2}}{2} \beta^{\frac{1}{p}}(p+1, p+2)\left[\left(\frac{2}{\pi}-\frac{4}{\pi^{2}}\right)\left|f^{\prime \prime}(a)\right|^{q}+\left(\frac{4}{\pi^{2}}\right)\left|f^{\prime \prime}(b)\right|^{q}\right]^{\frac{1}{q}} \\
& +\frac{(b-a)^{2}}{2} \beta^{\frac{1}{p}}(p+2, p+1)\left[\frac{4}{\pi^{2}}\left|f^{\prime}(a)\right|^{q}+\left(\frac{2}{\pi}-\frac{4}{\pi^{2}}\right)\left|f^{\prime}(b)\right|^{q}\right]^{\frac{1}{q}}
\end{aligned}
$$


where

$$
\begin{aligned}
& \int_{0}^{1} t^{p}(1-t)^{p+1} d t=\beta(p+1, p+2), \\
& \int_{0}^{1} t^{p+1}(1-t)^{p} d t=\beta(p+2, p+1) \\
& \int_{0}^{1}(1-t) \sin \frac{\pi t}{2} d t=\int_{0}^{1} t \cos \frac{\pi t}{2} d t=\frac{2}{\pi}-\frac{4}{\pi^{2}} \\
& \int_{0}^{1}(1-t) \cos \frac{\pi t}{2} d t=\int_{0}^{1} t \sin \frac{\pi t}{2} d t=\frac{4}{\pi^{2}} .
\end{aligned}
$$

This completes the proof of the theorem.

Remark 2.5. The inequality (2.2) is better than the inequality (2.1).

Proof. By using the properties

$$
\begin{aligned}
& \beta(p+1, p+2)=\beta(p+2, p+1) \\
& \beta(p+1, p+2)=\beta(p+1, p+1) \frac{p+1}{2(p+1)}
\end{aligned}
$$

and the concavity of the function $h:[0, \infty) \rightarrow \mathbb{R}, h(x)=x^{s}, 0<s \leq 1$, that is, if we use the property

$$
\frac{u^{s}+v^{s}}{2} \leq\left(\frac{u+v}{2}\right)^{s}
$$

we can write the right hand-side of the inequality (2.1) as follow:

$$
\begin{aligned}
& \frac{(b-a)^{2}}{2} \beta^{\frac{1}{p}}(p+1, p+2)\left[\left(\frac{2}{\pi}-\frac{4}{\pi^{2}}\right)\left|f^{\prime \prime}(a)\right|^{q}+\left(\frac{4}{\pi^{2}}\right)\left|f^{\prime \prime}(b)\right|^{q}\right]^{\frac{1}{q}}+\frac{(b-a)^{2}}{2} \beta^{\frac{1}{p}}(p+2, p+1)\left[\frac{4}{\pi^{2}}\left|f^{\prime \prime}(a)\right|^{q}+\left(\frac{2}{\pi}-\frac{4}{\pi^{2}}\right)\left|f^{\prime \prime}(b)\right|^{q}\right]^{\frac{1}{q}} \\
\leq & 2 \frac{(b-a)^{2}}{2} \beta^{\frac{1}{p}}(p+1, p+2)\left[\frac{\frac{2}{\pi}\left|f^{\prime \prime}(a)\right|^{q}+\frac{2}{\pi}\left|f^{\prime \prime}(b)\right|^{q}}{2}\right]^{\frac{1}{q}} \\
= & 2 \frac{(b-a)^{2}}{2}\left[\beta(p+1, p+1) \frac{p+1}{2(p+1)}\right]^{\frac{1}{p}}\left[\frac{\frac{2}{\pi}\left|f^{\prime \prime}(a)\right|^{q}+\frac{2}{\pi}\left|f^{\prime \prime}(b)\right|^{q}}{2}\right]^{\frac{1}{q}} \\
= & \frac{(b-a)^{2}}{2}\left(\frac{4}{\pi}\right)^{\frac{1}{q}} \beta^{\frac{1}{p}}(p+1, p+1) A^{\frac{1}{q}}\left(\left|f^{\prime \prime}(a)\right|^{q},\left|f^{\prime \prime}(b)\right|^{q}\right),
\end{aligned}
$$

which is the required result. This completes the proof of the Remark.

Theorem 2.6. Let $f: I \subseteq \mathbb{R} \rightarrow \mathbb{R}$ be a continuously two times differentiable function, let $a<b$ in $I$ and assume that $q \geq 1$. If the mapping $\left|f^{\prime \prime}\right|^{q}$ is trigonometrically convex function on interval $[a, b]$, then the following inequality holds for $t \in[0,1]$ :

$$
\left|\frac{f(a)+f(b)}{2}-\frac{1}{b-a} \int_{a}^{b} f(x) d x\right| \leq \frac{(b-a)^{2}}{2}\left(\frac{1}{6}\right)^{1-\frac{1}{q}}\left(\frac{8(4-\pi)}{\pi^{3}}\right)^{\frac{1}{q}} A^{\frac{1}{q}}\left(\left|f^{\prime \prime}(a)\right|^{q},\left|f^{\prime \prime}(b)\right|^{q}\right)
$$

Proof. From Lemma 2.1, power-mean integral inequality and trigonometrically convexity of $\left|f^{\prime \prime}\right|^{q}$, we have

$$
\begin{aligned}
\left|\frac{f(a)+f(b)}{2}-\frac{1}{b-a} \int_{a}^{b} f(x) d x\right| & \leq \frac{(b-a)^{2}}{2} \int_{0}^{1}|t||1-t|\left|f^{\prime \prime}(t a+(1-t) b)\right| d t \\
& \leq \frac{(b-a)^{2}}{2}\left(\int_{0}^{1} t(1-t) d t\right)^{1-\frac{1}{q}}\left(\int_{0}^{1} t(1-t)\left|f^{\prime \prime}(t a+(1-t) b)\right|^{q} d t\right)^{\frac{1}{q}} \\
& \leq \frac{(b-a)^{2}}{2}\left(\frac{1}{6}\right)^{1-\frac{1}{q}}\left(\int_{0}^{1} t(1-t)\left[\left(\sin \frac{\pi t}{2}\right)\left|f^{\prime \prime}(a)\right|^{q}+\left(\cos \frac{\pi t}{2}\right)\left|f^{\prime \prime}(b)\right|^{q}\right] d t\right)^{\frac{1}{q}} \\
& =\frac{(b-a)^{2}}{2}\left(\frac{1}{6}\right)^{1-\frac{1}{q}}\left(\left|f^{\prime \prime}(a)\right|^{q} \int_{0}^{1} t(1-t) \sin \frac{\pi t}{2} d t+\left|f^{\prime \prime}(b)\right|^{q} \int_{0}^{1} t(1-t) \cos \frac{\pi t}{2} d t\right)^{\frac{1}{q}} \\
& =\frac{(b-a)^{2}}{2}\left(\frac{1}{6}\right)^{1-\frac{1}{q}}\left(\frac{4(4-\pi)}{\pi^{3}}\left|f^{\prime \prime}(a)\right|^{q}+\frac{4(4-\pi)}{\pi^{3}}\left|f^{\prime \prime}(b)\right|^{q}\right)^{\frac{1}{q}} \\
& =\frac{(b-a)^{2}}{2}\left(\frac{1}{6}\right)^{1-\frac{1}{q}}\left(\frac{8(4-\pi)}{\pi^{3}}\right)^{\frac{1}{q}} A^{\frac{1}{q}}\left(\left|f^{\prime \prime}(a)\right|^{q},\left|f^{\prime \prime}(b)\right|^{q}\right)
\end{aligned}
$$

where

$$
\begin{aligned}
& \int_{0}^{1} t(1-t) d t=\frac{1}{6} \\
& \int_{0}^{1} t(1-t) \sin \frac{\pi t}{2} d t=\int_{0}^{1} t(1-t) \cos \frac{\pi t}{2} d t=\frac{4(4-\pi)}{\pi^{3}}
\end{aligned}
$$

This completes the proof of the theorem. 
Corollary 2.7. Under the assumption of Theorem 2.6 with $q=1$, we get the following the inequality:

$$
\left|\frac{f(a)+f(b)}{2}-\frac{1}{b-a} \int_{a}^{b} f(x) d x\right| \leq \frac{(b-a)^{2}}{2}\left(\frac{8(4-\pi)}{\pi^{3}}\right) A\left(\left|f^{\prime \prime}(a)\right|,\left|f^{\prime \prime}(b)\right|\right)
$$

Theorem 2.8. Let $f: I \subseteq \mathbb{R} \rightarrow \mathbb{R}$ be a continuously two times differentiable function, let $a<b$ in I and assume that $q \geq 1$. If the mapping $\left|f^{\prime \prime}\right|^{q}$ is trigonometrically convex function on interval $[a, b]$, then the following inequality holds for $t \in[0,1]$ :

$$
\begin{aligned}
\left|\frac{f(a)+f(b)}{2}-\frac{1}{b-a} \int_{a}^{b} f(x) d x\right| & \leq \frac{(b-a)^{2}}{2}\left(\frac{1}{12}\right)^{1-\frac{1}{q}}\left(\frac{32(\pi-3)}{\pi^{4}}\left|f^{\prime \prime}(a)\right|^{q}+\frac{4\left(24-4 \pi-\pi^{2}\right)}{\pi^{4}}\left|f^{\prime \prime}(b)\right|^{q}\right)^{\frac{1}{q}} \\
& +\frac{(b-a)^{2}}{2}\left(\frac{1}{12}\right)^{1-\frac{1}{q}}\left(\frac{4\left(24-4 \pi-\pi^{2}\right)}{\pi^{4}}\left|f^{\prime \prime}(a)\right|^{q}+\frac{32(\pi-3)}{\pi^{4}}\left|f^{\prime \prime}(b)\right|^{q}\right)^{\frac{1}{q}}
\end{aligned}
$$

Proof. From Lemma 2.1, improved power-mean integral inequality and trigonometrically convexity of $\left|f^{\prime \prime}\right|^{q}$, we have

$$
\begin{aligned}
\left|\frac{f(a)+f(b)}{2}-\frac{1}{b-a} \int_{a}^{b} f(x) d x\right| & \leq \frac{(b-a)^{2}}{2} \int_{0}^{1}|t||1-t|\left|f^{\prime \prime}(t a+(1-t) b)\right| d t \\
& \leq \frac{(b-a)^{2}}{2}\left(\int_{0}^{1} t(1-t)^{2} d t\right)^{1-\frac{1}{q}}\left(\int_{0}^{1} t(1-t)^{2}\left|f^{\prime \prime}(t a+(1-t) b)\right|^{q} d t\right)^{\frac{1}{q}} \\
& +\frac{(b-a)^{2}}{2}\left(\int_{0}^{1} t^{2}(1-t) d t\right)^{1-\frac{1}{q}}\left(\int_{0}^{1} t^{2}(1-t)\left|f^{\prime \prime}(t a+(1-t) b)\right|^{q} d t\right)^{\frac{1}{q}} \\
& \leq \frac{(b-a)^{2}}{2}\left(\frac{1}{12}\right)^{1-\frac{1}{q}}\left(\int_{0}^{1} t(1-t)^{2}\left[\left(\sin \frac{\pi t}{2}\right)\left|f^{\prime \prime}(a)\right|^{q}+\left(\cos \frac{\pi t}{2}\right)\left|f^{\prime \prime}(b)\right|^{q}\right] d t\right)^{\frac{1}{q}} \\
& +\frac{(b-a)^{2}}{2}\left(\frac{1}{12}\right)^{1-\frac{1}{q}}\left(\int_{0}^{1} t^{2}(1-t)\left[\left(\sin \frac{\pi t}{2}\right)\left|f^{\prime \prime}(a)\right|^{q}+\left(\cos \frac{\pi t}{2}\right)\left|f^{\prime \prime}(b)\right|^{q}\right] d t\right)^{\frac{1}{q}} \\
& =\frac{(b-a)^{2}}{2}\left(\frac{1}{12}\right)^{1-\frac{1}{q}}\left(\left|f^{\prime \prime}(a)\right|^{q} \int_{0}^{1} t(1-t)^{2} \sin \frac{\pi t}{2} d t+\left|f^{\prime \prime}(b)\right|^{q} \int_{0}^{1} t(1-t)^{2} \cos \frac{\pi t}{2} d t\right)^{\frac{1}{q}} \\
& +\frac{(b-a)^{2}}{2}\left(\frac{1}{12}\right)^{1-\frac{1}{q}}\left(\left|f^{\prime \prime}(a)\right|^{q} \int_{0}^{1} t^{2}(1-t) \sin \frac{\pi t}{2} d t+\left|f^{\prime \prime}(b)\right|^{q} \int_{0}^{1} t^{2}(1-t) \cos \frac{\pi t}{2} d t\right)^{\frac{1}{q}} \\
& =\frac{(b-a)^{2}}{2}\left(\frac{1}{12}\right)^{1-\frac{1}{q}}\left(\frac{32(\pi-3)}{\pi^{4}}\left|f^{\prime \prime}(a)\right|^{q}+\frac{4\left(24-4 \pi-\pi^{2}\right)}{\pi^{4}}\left|f^{\prime \prime}(b)\right|^{q}\right)^{\frac{1}{q}} \\
& +\frac{(b-a)^{2}}{2}\left(\frac{1}{12}\right)^{1-\frac{1}{q}}\left(\frac{4\left(24-4 \pi-\pi^{2}\right)}{\pi^{4}}\left|f^{\prime \prime}(a)\right|^{q}+\frac{32(\pi-3)}{\pi^{4}}\left|f^{\prime \prime}(b)\right|^{q}\right)^{\frac{1}{q}}
\end{aligned}
$$

where

$$
\begin{aligned}
& \int_{0}^{1} t(1-t)^{2} d t=\int_{0}^{1} t^{2}(1-t) d t=\frac{1}{12} \\
& \int_{0}^{1} t(1-t)^{2} \sin \frac{\pi t}{2} d t=\int_{0}^{1} t^{2}(1-t) \cos \frac{\pi t}{2} d t=\frac{32(\pi-3)}{\pi^{4}} \\
& \int_{0}^{1} t(1-t)^{2} \cos \frac{\pi t}{2} d t=\int_{0}^{1} t^{2}(1-t) \sin \frac{\pi t}{2} d t=\frac{4}{\pi^{4}}\left(24-4 \pi-\pi^{2}\right)
\end{aligned}
$$

This completes the proof of the theorem.

Remark 2.9. The inequality (2.4) is better than the inequality (2.3).

Proof. By using concavity of the function $h:[0, \infty) \rightarrow \mathbb{R}, h(x)=x^{s}, 0<s \leq 1$, we can write the right hand-side of the inequality (2.4) as follow:

$$
\begin{aligned}
& \frac{(b-a)^{2}}{2}\left(\frac{1}{12}\right)^{1-\frac{1}{q}}\left(\frac{32(\pi-3)}{\pi^{4}}\left|f^{\prime \prime}(a)\right|^{q}+\frac{4\left(24-4 \pi-\pi^{2}\right)}{\pi^{4}}\left|f^{\prime \prime}(b)\right|^{q}\right)^{\frac{1}{q}} \\
& +\frac{(b-a)^{2}}{2}\left(\frac{1}{12}\right)^{1-\frac{1}{q}}\left(\frac{4\left(24-4 \pi-\pi^{2}\right)}{\pi^{4}}\left|f^{\prime \prime}(a)\right|^{q}+\frac{32(\pi-3)}{\pi^{4}}\left|f^{\prime \prime}(b)\right|^{q}\right)^{\frac{1}{q}} \\
& \leq 2 \frac{(b-a)^{2}}{2}\left(\frac{1}{12}\right)^{1-\frac{1}{q}}\left(\frac{4(4-\pi)}{\pi^{3}} \frac{\left|f^{\prime \prime}(a)\right|^{q}+\left|f^{\prime \prime}(b)\right|^{q}}{2}\right)^{\frac{1}{q}} \\
& =2 \frac{(b-a)^{2}}{2}\left(\frac{1}{12}\right)^{1-\frac{1}{q}}\left(\frac{4(4-\pi)}{\pi^{3}}\right)^{\frac{1}{q}} A^{\frac{1}{q}}\left(\left|f^{\prime \prime}(a)\right|^{q},\left|f^{\prime \prime}(b)\right|^{q}\right) \\
& =\frac{(b-a)^{2}}{2}\left(\frac{1}{6}\right)^{1-\frac{1}{q}}\left(\frac{8(4-\pi)}{\pi^{3}}\right)^{\frac{1}{q}} A^{\frac{1}{q}}\left(\left|f^{\prime \prime}(a)\right|^{q},\left|f^{\prime \prime}(b)\right|^{q}\right),
\end{aligned}
$$


which is the required result. This completes the proof of the Remark.

Corollary 2.10. Under the assumption of Theorem 2.8 with $q=1$, we get the following the inequality:

$$
\left|\frac{f(a)+f(b)}{2}-\frac{1}{b-a} \int_{a}^{b} f(x) d x\right| \leq \frac{(b-a)^{2}}{2} \frac{8(4-\pi)}{\pi^{3}} A\left(\left|f^{\prime \prime}(a)\right|,\left|f^{\prime \prime}(b)\right|\right) .
$$

\section{References}

[1] S.S. Dragomir and R.P. Agarwal, Two inequalities for differentiable mappings and applications to special means of real numbers and to trapezoidal formula, Appl. Math. Lett. 11(5) (1998), 91-95.

[2] S.S. Dragomir, Refinements of the Hermite-Hadamard integral inequality for log-convex functions, Aust. Math. Soc. Gaz. 28(3) (2001), 129-134.

[3] S.S. Dragomir and C.E.M. Pearce, Selected Topics on Hermite-Hadamard Inequalities and Its Applications, RGMIA Monograph 2002.

[4] S.S. Dragomir, J. Pečarić and LE.Persson, Some inequalities of Hadamard Type, Soochow Journal of Mathematics, 21(3) (2001), pp. 335-341.

[5] J. Hadamard, Etude sur les proprietes des fonctions entieres en particulier d'une fonction consideree par Riemann, J. Math. Pures Appl. 58 (1893), $171-215$.

[6] İ. İşcan, New refinements for integral and sum forms of Hölder inequality, Journal of Inequalities and Applications, (2019) 2019:304

[7] H. Kadakal, Hermite-Hadamard type inequalities for trigonometrically convex functions, Scientific Studies and Research. Series Mathematics and Informatics, 28(2) (2018), 19-28.

[8] M. Kadakal, İ. İşcan, H. Kadakal and K. Bekar, On improvements of some integral inequalities, Researchgate, DOI: 10.13140/RG.2.2.15052.46724, Preprint, January 2019.

[9] M. Kadakal, H. Kadakal and İ. İşcan, Some new integral inequalities for n-times differentiable s-convex functions in the first sense, Turkish Journal of Analysis and Number Theory, 5(2) (2017), 63-68.

[10] S. Maden, H. Kadakal, M. Kadakal and İ. İşcan, Some new integral inequalities for n-times differentiable convex and concave functions, Journal of Nonlinear Sciences and Applications, 10(12) (2017), 6141-6148.

[11] F. Usta, H. Budak and M. Z. Sarıaya, Montgomery identities and Ostrowski type inequalities for fractional integral operators, Revista de la Real Academia de Ciencias Exactas, Físicas y Naturales. Serie A. Matemáticas, 113(2) (2019), 1059-1080

[12] F. Usta, H. Budak and M. Z. Sarkaya, Some New Chebyshev Type Inequalities Utilizing Generalized Fractional Integral Operators, AIMS Mathematics, $\mathbf{5}(2)(2020)$ 1147-1161.

[13] F. Usta, H. Budak, M. Z. Sarıkaya and E. Set, On generalization of trapezoid type inequalities for s-convex functions with generalized fractional integral operators, Filomat, 32(6) (2018), 2153-2171.

[14] S. Varošanec, On h-convexity, J. Math. Anal. Appl. 326 (2007) 303-311.

[15] G. Zabandan, A new refinement of the Hermite-Hadamard inequality for convex functions, J. Inequal. Pure Appl. Math. 10(2) (2009), Article ID 45. 